\title{
Configurational Characteristics of Poly(oxy-1,1-dimethylethylene). III. Configuration-Dependent Properties of the Polymer Chain
}

\author{
Akihiro ABE, Isao ANDO, Kazuhiro KATO, \\ and Ichitaro UEMATSU \\ Department of Polymer Chemistry, Tokyo Institute of Technology, \\ Meguro-ku, Tokyo 152, Japan.
}

(Received June 13, 1981)

\begin{abstract}
The configurational characteristics of poly(oxy-1,1-dimethylethylene) (PODME) were estimated by calculation based on the information acquired through the dipole moment and NMR analyses of 1,2-dimethoxy-2-methylpropane, and, in part, of PODME as presented in the preceding papers. By using the set of conformational energy parameters so established,. the characteristic ratio $\left\langle r^{2}\right\rangle_{0} / n l^{2}$ and the dipole moment ratio $\left\langle\mu^{2}\right\rangle / n m^{2}$ were evaluated. These results were compared with those for poly(oxyethylene) and poly(oxypropylene) studied previously. The configurational entropy calculated for the temperature equivalent to the melting point $\left(175^{\circ} \mathrm{C}\right)$ of the polymer is $4.1 \mathrm{cal} \mathrm{mol}^{-1} \mathrm{deg}^{-1}$. It was pointed out that a fairly large value of the enthalpy of fusion should be required to explain this comparatively high melting temperature of PODME, unless the correction term for the entropy of fusion emerging from the volume change on melting is very small.
\end{abstract}

KEY WORDS Poly(oxy-1,1-dimethylethylene) / 1,2-Dimethoxy-2-methylpropane / Unperturbed Dimension / Dipole Moment / Configurational Entropy / Poly(oxyethylene) / Poly(oxypropylene) /

Geometrical parameters and conformational energies established in the preceding papers ${ }^{1,2}$ (referred to as papers I and II) enable us to evaluate various configuration-dependent properties of the poly(oxy-1,1-dimethylethylene) (PODME) chain which are otherwise quite inaccessible: PODME is a crystalline polymer with a high melting point $\left(175^{\circ} \mathrm{C}\right)^{3}$ and only poorly soluble in conventional organic solvents. The results of these calculations will be compared with those of other polymers having related structures such as poly(oxyethylene) ${ }^{4}$ (POE) and poly(oxypropylene) ${ }^{5}$ (POP). The polymer chains mentioned above can be represented by a general formula $\left\{\mathrm{CH}_{2} \mathrm{C}\left(\mathrm{R}_{1}\right)\left(\mathrm{R}_{2}\right) \mathrm{O}\right\}_{x}$, where $\mathrm{R}_{1}$ and $\mathrm{R}_{2}$ may be a proton atom or a methyl group depending upon the polymer system. The conformational flexibility of a given (skeletal) bond varies with the degree of methyl substitution. As is evident from the results presented in papers I and II, the bond rotation around the skeletal $\mathrm{C}-\mathrm{C}$ bond tends to be more restricted in the order POE, POP, and PODME, while the number of conformers per- mitted to the neighboring $C\left(R_{1}\right)\left(R_{2}\right)-O$ bond becomes more proliferated as $\mathrm{R}$ becomes bulkier. Accordingly, the molar configurational partition function of PODME obtained in the preceding work ${ }^{2}$ is similar in magnitude to that of $\mathrm{POE}^{4}$ and POP. ${ }^{5}$

The most stable conformations found in the crystalline state for the moiety $\mathrm{O}-\mathrm{CH}_{2}-$ $\mathrm{C}\left(\mathrm{R}_{1}\right)\left(\mathrm{R}_{2}\right)-\mathrm{O}$ are $\left(t g^{ \pm} t\right),(t t t)$, and $\left(t^{ \pm} t t^{\mp}\right)$, respectively, for POE $^{6}{ }^{6}$ isotactic POP, ${ }^{7}$ and PODME. $^{8}$ For a better understanding of the characteristic feature at fusion, configurational contribution to the entropy change on melting will be estimated.

\section{GEOMETRICAL PARAMETERS AND CONFORMATIONAL ENERGIES}

Bond lengths and bond angles required in the description of the skeletal chain of PODME were taken from Table II of paper I, in which geometrical parameters used in the analysis of DMMP are 
summarized. Unless otherwise noted, we shall adopt $\angle \mathrm{COC}=118^{\circ}$ for all ether linkages except the one associated with bonds 1 and 2, for which $\angle \mathrm{COC}=111.5^{\circ}$ was employed. The other bond lengths and bond angles are comparable to those used in the treatment of $\mathrm{POE}^{4}, \mathrm{POP}^{5}$ and their analogs. Configurational partition function $Z$ for a given chain may be calculated ${ }^{9}$ by using eq $3-8$ of paper I. The conformational energy parameters required here are given in Table III of paper I. In accordance with the experimental observations reported in papers I and II, the value of $E_{\sigma}$ is taken to be $0.5 \mathrm{kcal} \mathrm{mol}^{-1}$.

Based on the results of the conformational energy calculations presented in paper $I$, rotational isomeric states $t, g^{+}$, and $g^{-}$were taken to occur at the regularly staggered position, i.e., $\phi_{t}=0$ and $\phi_{g \pm}= \pm(2 / 3) \pi$, for bonds like a and b (cf. eq $6-8$ and Figure 1 of paper I). The gauche conformations for bond $\mathrm{c}$ involve prohibitingly severe steric conflicts between the two $>\mathrm{C}\left(\mathrm{CH}_{3}\right)_{2}$ groups: a value of $\phi_{t}=0$ was accordingly assigned to the remaining trans state.

\section{THEORETICAL ESTIMATION OF THE UNPERTURBED DIMENSION, DIPOLE MOMENT, AND CONFIGURATIONAL ENTROPY}

Calculations were carried out ${ }^{9}$ for chains such as $\mathrm{CH}_{3} \mathrm{O}+\mathrm{CH}_{2} \mathrm{C}\left(\mathrm{CH}_{3}\right)_{2} \mathrm{O}_{x} \mathrm{CH}_{3}$ for the temperature of $25^{\circ} \mathrm{C}$. As is now customary, ${ }^{9}$ the calculated values of the unperturbed dimensions and dipole moments were expressed as the characteristic ratios $\left\langle r^{2}\right\rangle_{0} / n l^{2}$ and $\left\langle\mu^{2}\right\rangle / n m^{2}$, respectively, where $n$ is the number of skeletal bonds, and $l^{2}$ and $n^{2}$ are the averaged values of the squares of their lengths and dipole moments, respectively. The asymptotic behaviors estimated for these ratios with the degree of polymerization $x$ are given in Figures $1 \mathrm{a}$ and $1 \mathrm{~b}$. Also shown for comparison in these figures are the results obtained by using $\angle C O C=111.5^{\circ}$ for all the ether linkages in the chain, with the other parameters kept invariable. Such an alteration causes a decrease of as much as $c a .5 \%$ in $\left\langle r^{2}\right\rangle_{0} / n l^{2}$ and an increase of $c a .10 \%$ in $\left\langle\mu^{2}\right\rangle / n m^{2}$, but generally the effect is only trivial. Both $\left\langle r^{2}\right\rangle_{0} / n l^{2}$ and $\left\langle\mu^{2}\right\rangle / n m^{2}$ reach quite rapidly their asymptotic values for $x \rightarrow \infty$ : e.g. at $x \cong 10$, the former ratio comes into the range within $10 \%$ of the limiting

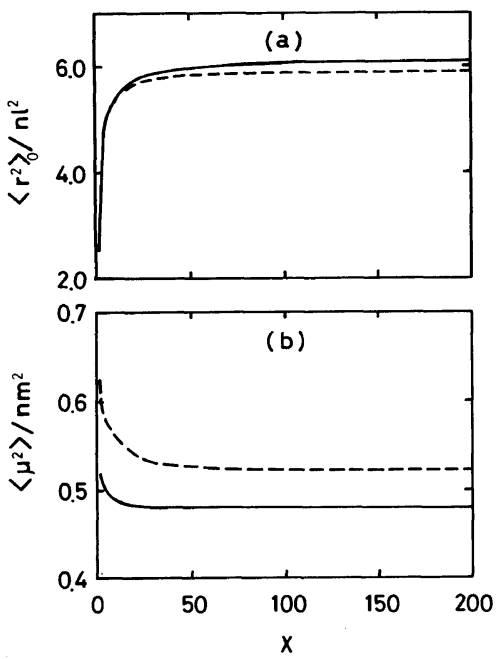

Figure 1. Dependence of the characteristic ratios (a) $\left\langle r^{2}\right\rangle_{0} / n l^{2}$ and (b) $\left\langle\mu^{2}\right\rangle / n m^{2}$ on the degree of polymerization $x$ for the PODME chain $\left(T=25^{\circ} \mathrm{C}\right)$. The solid curves indicate the results obtained by using $\angle \mathrm{COC}=118^{\circ}$ for the internal ether linkages as established in this work. The broken curves represent the results derived by adopting a value of $111.5^{\circ}$ for all the COC angles.

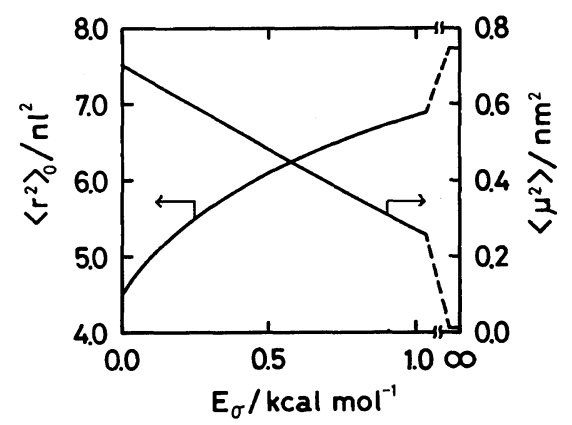

Figure 2. The characteristics ratios $\left\langle r^{2}\right\rangle_{0} / n l^{2}$ and $\left\langle\mu^{2}\right\rangle / n m^{2}$ for the PODME chains having $x=200$ ( $n=602)$ calculated as a function of $E_{\sigma}$ for the temperature of $25^{\circ} \mathrm{C}$. The other statistical weight parameters were kept invariable (see text).

value, while the latter finds inself within a few $\%$ of its limit.

In accordance with the procedure adopted previously in the treatment of polyoxide chains, ${ }^{4,5}$ variations of $\left\langle r^{2}\right\rangle_{0} / n l^{2}$ and $\left\langle\mu^{2}\right\rangle / n m^{2}$ with the conformational energy parameter $E_{\sigma}$ were investigated over the range $E_{\sigma}>0$ (Figure 2). As $E_{\sigma}$ increases, the trans conformation tends to be more stabilized 
around the skeletal $\mathrm{C}-\mathrm{C}$ bond. The value of $\left\langle r^{2}\right\rangle_{0} / n l^{2}$ increases from 5.0 to 7.0 over the range $E_{\sigma}=0$ to $1.0 \mathrm{kcal} \mathrm{mol}^{-1}$, beyond which the ratio varies very slowly toward its limiting value 7.5 . At this limit, the flexibility of the PODME chain arises solely from the bond rotation around the $\mathrm{C}\left(\mathrm{CH}_{3}\right)_{2}-\mathrm{O}$ bond. The value of $\left\langle\mu^{2}\right\rangle / \mathrm{nm}^{2}$ decreases rapidly with an increase of $E_{\sigma}$. Although the chain is still quite flexible around the aforementioned bond, the ratio becomes nearly zero at the limit $E_{\sigma}=\infty$.

The temperature coefficients calculated for a polymer chain with $x=200$ by using the parameters set forth above are as follows: $10^{3} \mathrm{~d} \ln \left\langle r^{2}\right\rangle_{0} / \mathrm{d} T=$ $-0.87 \mathrm{~K}^{-1}$ and $10^{3} \ln \left\langle\mu^{2}\right\rangle / \mathrm{d} T=0.81 \mathrm{~K}^{-1}$ (at $25^{\circ} \mathrm{C}$ )

Following the treatment previously employed in the analysis of POE and related polymers, ${ }^{10}$ the configurational partition function for an internal monomeric residue

$$
z=Z^{1 / x}
$$

and the configurational entropy

$$
S_{\text {conf }}=R[\ln z+\mathrm{d} \ln z / \mathrm{d} \ln T]
$$

were evaluated. The values obtained for a chain of 200 units at the temperature equivalent to the melting point of the polymer are as follows: $z=5.6$ and $S_{\text {conf }}=4.1 \mathrm{cal} \mathrm{mol}^{-1} \mathrm{deg}^{-1}$. The configurational entropy obtained in this manner corresponds to the entropy of fusion at constant volume. However, no relevant experimental data are yet available.

\section{COMPARISON WITH POE AND POP}

Extra stabilization energies $(\Delta E)$ associated with the gauche oxygen effect have been defined previously as the difference between the conformational energies $\left(E_{\text {calcd }}\right)$ calculated by using semiempirical expressions and those $\left(E_{\text {expt1 }}\right)$ derived from the analysis of the experimental data such as $\left\langle r^{2}\right\rangle_{0} / n l^{2},\left\langle\mu^{2}\right\rangle / n m^{2}$, and bond conformations. ${ }^{4,5}$ The values of $\Delta E$ thus established for the gauche conformations about the $\mathrm{C}-\mathrm{C}$ bonds are summarized in Tabel I for the aforementioned polymer systems. It should be noted here that nearly the same values of $E$ 's, and thus $\Delta E$ 's have been obtained from the analysis of the corresponding monomeric model compounds (dimethoxy derivatives) $)^{1,2,4,5,11}$ in all these cases, indicating that the results shown in the table are inherent in the chemical structure and therefore valid regardless of chain length. In Table I, the magnitude of $\Delta E$ varies from $1.0 \mathrm{kcal} \mathrm{mol}^{-1}$ (for POE) to zero (for PODME). The two gauche states around the skeletal $\mathrm{C}-\mathrm{C}$ bond of POP are unequivalent to each other with regard to steric environment. It is interesting to note here that the value of $\Delta E$ for this polymer falls into either of these two extremes: one $\left(\Delta E_{\alpha}=0.7\right.$ $\mathrm{kcal} \mathrm{mol}^{-1}$ ) close to the value of POE and the other $\left(\Delta E_{\beta}=0.2 \mathrm{kcal} \mathrm{mol}^{-1}\right)$ to that of PODME, depending on the steric requirements of the conformation. Further penetration into this problem has been attempted within the framework of the molecular orbital theory. ${ }^{12}$ The results show success only in certain limited cases, however.

Shown in Table II are the results of calculations

Table I. gauche Oxygen effects associated with gauche conformations around the skeletal $\mathrm{C}-\mathrm{C}$ bond for the POE, POP, and PODME chains ${ }^{\mathrm{a}}$

\begin{tabular}{lrccc}
\hline & & \multicolumn{2}{c}{ POP $^{\mathrm{c}}$} & PODME \\
\cline { 3 - 5 } & & gauche $\alpha^{\mathrm{d}}$ & gauche $\beta^{\mathrm{e}}$ & \\
\hline$E_{\text {calcd }}$ & 0.6 & 0.4 & 0.6 & 0.5 \\
$E_{\text {expl }}$ & -0.4 & -0.3 & 0.4 & 0.5 \\
$\Delta E$ & 1.0 & 0.7 & 0.2 & 0.0 \\
\hline
\end{tabular}

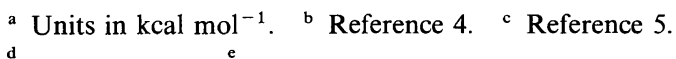<smiles>CC1CCC2OC1CC2O</smiles> 
Table II. Characteristic ratio $\left\langle r^{2}\right\rangle_{0} / n l^{2}$, dipole moment ratio $\left\langle\mu^{2}\right\rangle / n m^{2}$, and their temperature coefficients for the POE, POP, and PODME chains

\begin{tabular}{|c|c|c|c|c|}
\hline \multirow{2}{*}{ Polymer } & \multirow{2}{*}{$\left\langle r^{2}\right\rangle_{0} / n l^{2}$} & $10^{3} \mathrm{~d} \ln \left\langle r^{2}\right\rangle_{0} / \mathrm{d} T$ & \multirow{2}{*}{$\left\langle\mu^{2}\right\rangle / n m^{2}$} & \multirow{2}{*}{$\frac{10^{3} \mathrm{~d} \ln \left\langle\mu^{2}\right\rangle / \mathrm{d} T}{\mathrm{~K}^{-1}}$} \\
\hline & & $\mathbf{K}^{-1}$ & & \\
\hline $\mathrm{POE}^{\mathrm{a}}$ & 5.0 & 0.14 & 0.49 & 2.9 \\
\hline $\begin{array}{l}\text { Isotactic }^{\mathrm{b}} \\
\text { POP }\end{array}$ & 6.1 & -1.59 & 0.50 & 0.43 \\
\hline PODME & 6.1 & -0.87 & 0.48 & 0.81 \\
\hline
\end{tabular}

${ }^{\text {a }}$ Reference 4. ${ }^{\mathrm{b}}$ Reference 5.

for $\left\langle r^{2}\right\rangle_{0} / n l^{2}$ and $\left\langle\mu^{2}\right\rangle / n m^{2}$, and their temperature coefficients for $\mathrm{POE}^{4}, \mathrm{POP}^{5}$, and PODME. These ratios were derived by taking statistical mechanical averages over all configurations of the chain. The conformational characteristics of individual chains such as those discussed above do not manifest themselves explicitly in the final results.

The value of configurational entropy calculated above may be compared with those of other polymers as tabulated in a previous paper. ${ }^{10}$ The value of $S_{\text {conf }}=5.0 \mathrm{cal} \mathrm{mol}^{-1} \mathrm{deg}^{-1}$ given for POE in this table (Table IV of ref 10 ) should be substituted by a newer one $\left(5.3 \mathrm{cal} \mathrm{mol}^{-1} \mathrm{deg}^{-1}\right)$ as a more reliable value $^{13}$ of the melting temperature $\left(79 \pm 1^{\circ} \mathrm{C}\right)$ is adopted for this polymer. The value of the configurational entropy of PODME is similar in magnitude to that $\left(4.0 \mathrm{cal} \mathrm{mol}^{-1} \mathrm{deg}^{-1}\right)$ of POP.

\section{CONCLUDING REMARKS}

The value of $S_{\text {conf }}=4.1 \mathrm{cal} \mathrm{mol}^{-1} \mathrm{deg}^{-1}$ for PODME calculated above remains nearly invariable even when $E_{\gamma}=0.5 \mathrm{kcal} \mathrm{mol}^{-1}$ is tentatively assigned to the rotation around the $\mathrm{C}\left(\mathrm{CH}_{3}\right)_{2}-\mathrm{O}$ bond ( $c f$. Table III of paper I): with an increase in $E_{\gamma}$, the value of $z$, thus the first term of the righthand side of eq $2(\ln z)$, decreases while the contribution from the second term $(\mathrm{d} \ln z / \mathrm{d} \ln T)$ increases as compensation. Unless the correction term in the entropy of fusion for the volume change on melting is very small, ${ }^{14} \mathrm{a}$ fairly large value of the enthalpy of fusion should be required to explain the comparatively high melting temperature of PODME.

Crystallographic studies of PODME have been carried out by Kaji and Sakurada, ${ }^{8}$ who reported that the polymer chain takes a trans zigzag conformation, but distorted somewhat from the perfect planarity, in the crystalline state. This conformation has been represented by $t^{ \pm} t t^{\mp}$ for the sequence $\mathrm{O}-\mathrm{CH}_{2}-\mathrm{C}\left(\mathrm{CH}_{3}\right)_{2}-\mathrm{O}$, where the rotational minima for the $\mathrm{C}-\mathrm{O}$ and $\mathrm{O}-\mathrm{C}$ bonds are displaced by $c a$. $\pm 27^{\circ}$ from the regular trans position. Thus, the chain has a $1 / 2$ helix axis. The crystalline unit cell is orthorhombic, comprising four monomeric units. As these authors pointed out, ${ }^{8}$ PODME is quite similar to $\mathrm{POP}^{7}$ and poly(thiopropylene) ${ }^{15}$ not only in chain conformation but also in crystalline arrangement. No particular reasons may be noticed in this arrangement why the enthalpy of fusion should be enhanced. ${ }^{16}$ In fact, a preliminary study by Ikawa ${ }^{17}$ yielded a value of the enthalpy of fusion $\Delta H_{\mathrm{f}}=2.2-2.5 \mathrm{kcal} \mathrm{mol}^{-1}$, and accordingly, the corresponding entropy $\Delta S_{\mathrm{f}}=4.6-5.3 \mathrm{cal} \mathrm{mol}^{-1}$ $\mathrm{deg}^{-1}$ for the equilibrium melting temperature of $202^{\circ} \mathrm{C}$. This value of $\Delta H_{\mathrm{f}}$ is only slightly higher than that $\left(\Delta H_{\mathrm{f}}=2.0 \mathrm{kcal} \mathrm{mol}^{-1}\right)$ of POP. ${ }^{18}$ These results are therefore somewhat inconsistent with those derived above. Further investigation is now being carried out on this subject.

Acknowledgment. We are grateful to the Asahi Glass Foundation for Industrial Technology for financial support.

\section{REFERENCES}

1. K. Kato, K. Araki, and A. Abe, Polym. J., 13, 1055 (1981).

2. I. Ando, K. Sato, K. Kato, and A. Abe, Polym. J., 13, 1063 (1981).

3. J. Boor and R. S. Bauer, J. Appl. Polym. Sci., 18, 3699 (1974).

4. A. Abe and J. E. Mark, J. Am. Chem. Soc., 98, 6468 (1976).

5. A. Abe, T. Hirano, and T. Tsuruta, Macromolecules, 


\section{Configurational Characteristics of PODME. III.}

12, 1092 (1979).

6. Y. Takahashi and H. Tadokoro, Macromolecules, 6, 672 (1973).

7. M. Cesari, G. Perego, and W. Marconi, Makromol. Chem., 94, 194 (1966).

8. K. Kaji and I. Sakurada, Makromol. Chem., 148, 261 (1971); I. Sakurada and K. Kaji, J. Polym. Sci., C31, 57 (1970).

9. P. J. Flory, "Statistical Mechanics of Chain Molecules," Interscience, New York, 1969.

10. A. Abe, Macromolecules, 13, 546 (1980).

11. V. Viti, P. L. Indovina, F. Podo, L. Radics, and G. Nemethy, Mol. Phys., 27, 541 (1974).
12. T. Hirano, Polym. Prepr., Jpn., 29, 542 (1980); Y. Sasanuma, I. Ando, and A. Abe, unpublished results.

13. L. Mandelkern, private communication.

14. L. Mandelkern, "Crystallization of Polymers," McGraw-Hill, New York, 1964.

15. H. Sakakibara, Y. Takahashi, H. Tadokoro, P. Sigwalt, and N. Spassky, Macromolecules, 2, 515 (1969).

16. H. Tadokoro, "Structure of Crystalline Polymers," Wiley-Interscience, New York, 1979.

17. T. Ikawa, private communication.

18. G. Allen, C. Booth, M. N. Jones, D. J. Marks, and W. D. Taylor, Polymer, 5, 547 (1964). 